\section{Fifth "dark" force completely change our understanding of the universe}

\author{
Robert Skopec* \\ Researcher Analyst, Dubnik, Slovakia
}

\section{Abstract}

For something that's literally as old as the universe, dark matter doesn't get much attention outside scientific circles. Maybe that's because, other than a short-lived SyFy series and a lateperiod Randy Newman album, this nebulous star stuff has had a tough time breaking the popculture barrier. But the truth is that today, dark matter has never mattered more. Our own Milky Way is embedded in a massive cloud of it, we're looking for its interactions deep inside the earth, and there are whole galaxies without it. So what is dark matter, anyway? Why can't scientists get enough of the stuff, even though they can't actually find it? What deep, dark secrets does it hold? And could it ultimately shape the future of life as we know it?

\section{Introduction}

The history of dark matter shows just how elusive this star stuff can be. Physicists first theorized the existence of dark matter in 1933, partly because equations showed that there wasn't enough observable matter in galaxies to keep them from disintegrating, and observed rotational speeds of galaxies didn't fit the expected results from standard physics models. Further research largely waited until the 1970 s, when better scientific instruments, from receivers to gamma radiationdetecting space telescopes, let astronomers and physicists confirm the earlier calculations and observations. Powerful radio telescopes also offered clues like gravitational lensing (where matter causes light to bend between its source and the observer) and strongly suggested that there was a kind of matter out there we could detect, but not see [1-3]. "Everything you can see, everything you feel, everything you're made up of, only makes up $5 \%$ of the universe, and the rest is this dark stuff...and we have no idea what it is," says Rebecca Leane, a theoretical physicist at MIT. Leane's Ph.D. dissertation was on the phenomenology of dark matter. Physicists estimate some $27 \%$ of the total universe is dark matter and the rest (68\%) is a similarly shadowy phenomenon called dark energy. What makes dark matter so mysterious? [4-6]. "The big thing about it is that we can't see it; it doesn't interact with light," says Ethan Brown, an Assistant Professor of Physics at Rensselaer Polytechnic Institute. (The photo at the top of this article is a composite image from optical and $\mathrm{x}$-ray telescopes where the blue shading depicts the likely dark matter, even though it doesn't show up directly in the images).

\section{More Information}

*Address for Correspondence: Robert Skopec, Researcher Analyst, Dubnik, Slovakia, Email: zxcbnvm7@gmail.com

\author{
Submitted: 26 June 2019 \\ Approved: 26 July 2019 \\ Published: 29 July 2019
}

How to cite this article: Skopec R. Fifth "dark" force completely change our understanding of the universe. Arch Cancer Sci Ther. 2019; 3: 035-041.

DOI: dx.doi.org/10.29328/journal.acst.1001006

Copyright: @ 2019 Skopec R. This is an open access article distributed under the Creative Commons Attribution License, which permits unrestricted use, distribution, and reproduction in any medium, provided the original work is properly cited

Keywords: Dark matter; Dark energy; Radioactive decay; Neutrinoless double beta decay; Majorana fermion; Superflares; Coronal mass ejection; NASA Kepler space telescope; European Space Agency's Gaia spacecraft; Gravitational lensing; Gamma radiation-detecting space telescopes

Check for updates

Broadly, we can measure matter and energy in the universe by observing it in one of four interactions:

- With electromagnetic radiation (a.k.a. light)

- Through gravitational effects

- With other matter through the strong nuclear force, which holds matter together

- With the weak nuclear force, or the interaction of subatomic particles that's responsible for radioactive decay

Dark matter eludes most of those observations because it doesn't appear to interact with standard matter at all, except through gravity. But that hasn't stopped physicists from ruling out other methods [7-9]. One of Brown's areas of study is trying to capture dark matter interactions with normal matter in the form of liquid xenon isotopes. Xenon-124 has a half life roughly a trillion times longer than the age of the universe. Massive vats of the stuff are tucked deep into boreholes in the earth's crust to limit background noise like electromagnetic radiation that could interfere with measurements. Only dark matter and certain subatomic particles like muons and neutrinos can pass through the thousands of feet of dense rock. So it's a very "quiet" room, where-theoretically-only Xenon-124's exceptionally slow natural radioactive decay, or interactions with muons, neutrinos, or dark matter could 
cause some kind of change in the isotope. If a subatomic particle of dark matter knocks out an electron from the Xenon-124, the thinking goes, the Xenon1T experiment will see it. While dark matter scientists haven't actually detected direct interactions with the elusive subatomic particles yet, they've certainly made some other interesting observationsincluding the decay of xenon-124, only the rarest event ever recorded in human history.

\section{So what is dark matter?}

We know more about what dark matter isn't than what it is. For starters, it isn't dark energy. That's some kind of energy for which the evidence is also indirect, but likely exists because the universe is expanding at an increasing rate of speed, which defies the laws of physics of normal matter and energy. And dark matter isn't antimatter, either, which is normal matter composed of subatomic particles that have an exact opposite charge to matter. When antimatter and matter collide, the annihilation produces bursts of gamma rays. Dark matter can also produce gamma rays when it and its counterpart, dark antimatter, collide to produce standard matter. And finally, dark matter isn't just a different class of the three families of ordinary matter like hadrons, leptons, or bosons, the latter two of which were formerly theoretical, but have finally been directly observed in particle accelerators and don't behave like we expect [10-12]. Leptons and bosons do give us a hint to follow, however. Dark matter appears to be a form of matter made up of an entirely different class or classes of subatomic particle. One of the most promising is called a WIMP: a weakly interacting massive particle.

WIMPs, despite their puny name, are thought to have a mass as much as a thousand times more than standard matter's protons. And the way that WIMPs are theorized to work fits neatly with calculations of how much dark matter there must be in the universe, says Leane. This is called the WIMP Miracle. But WIMPs are far from the only theory in play. There are also primordial black holes, which are essentially small black holes left over from the Big Bang. However, we haven't observed gravitational microlensing from them, so that rules out some masses of primordial black holes as possible dark matter. Then there are theorized particles like SIMPs and axions-and countless other potential clues. "There are more theories out there now than I'll ever understand," Brown admits. Naturally, it can be pretty annoying to do research when you can't actually observe something you think exists, or isn't always there. Researchers at Yale, for example, have found two galaxies that don't have any dark matter at all.

"It's hard to point out just one solution for how these might have formed," says Shany Danieli, a doctoral student at Yale who co-authored two of the studies. "At the beginning, we thought maybe it was just some kind of anomaly, but now we found a second galaxy." The research points to some fascinating possibilities for how dark matter functions in the universe: that dark matter interacts with normal matter via a mechanism that we don't yet know-a so-called "dark force," or fifth force in the universe [6,11]. Another idea is that dark matter does interact via more of the known forces than just gravity, but does so at such a tiny interaction strength that we simply don't have the means yet to reliably detect the signals. In other words, the science is far from conclusive.

\section{What dark matter means}

Why, then, are physicists so focused on unraveling the mystery of dark matter? "The work of particle physics the past 50 years has been to break down the universe to its smallest components," says RPI's Brown. Right now, dark matter doesn't fit certain understandings of how the universe works, in particular the standard model of particle physics. "When we understand what dark matter is, and how it behaves, that's a huge step to understand the fundamental underpinnings of the universe," Brown says. "We can answer questions like how did the universe develop to what it is today?" Plus, fundamental particle physics, including the search for dark matter, has already produced real technological gains. Many of the detection tools used in the field are highly applicable to other areas like medical imaging or nuclear security [13-15].

Leane points out that the internet was created in part because particle physicists at CERN wanted to find new ways to share data with each other. GPS, meanwhile, relies in some measure on Einstein's theory of general relativity, which explains how gravity curves space and time, says Danieli. We can't begin to know what might emerge from our study of dark matter, but consider an analogue from the study of conventional matter, which yielded the most fundamental technology that allows us to do practically anything in modern life. Without J.J. Thomson's discovery of the electron in 1897, we wouldn't even have electricity, much less computers and an internet powered by it. So while we still don't know much about dark matter today, it could very well change the way we live tomorrow [11].

\section{Dark matter in the cosmos}

Our universe may have suddenly inflated from a tiny point during the Big Bang (now estimated to have occurred around 13.7 billion years ago -- more) to create dark energy (74\%) and dark matter (22\%), as well as a small amount (4\%) of ordinary matter in the form of electrons and quarks in a superhot plasma (more on the proportion of matter in the "Cinderella Universe" model from SDSS). Small clouds of dark matter have been coalescing and gradually merging together through gravitational attraction ever since that incredible explosion. Although dark matter makes up as much as $85 \%$ of all the matter in the universe, astronomers do not actually know what it is composed of (see a discussion of candidates). When a sufficient amount of dark matter has gathered, it attracts ordinary matter (mostly hydrogen and helium gas) to form stars that may eventually form a luminous galaxy at the 
core. While particles of ordinary matter readily interact with one another and, if electrically charged, with electromagnetic radiation, dark matter is comprised of particles that do not react with such radiation, although dark matter interacts gravitationally just like ordinary matter (Christopher J. Conselice, 2007).

On January 7, 2007, a team of astronomers announced that they had used observations obtained with the Hubble Space Telescope to create a three-dimensional map of the large-scale distribution of dark matter in part of the universe. This map provides the best evidence to date that normal matter, largely in the form of galaxies, accumulates along the densest concentrations of dark matter. It depicts a loose network of dark-matter filaments that grew over time and intersect in massive structures at the locations of clusters of galaxies. Stretching halfway back in time to the beginning of the universe, the map shows how dark matter has grown increasingly "clumpy" as it collapses under gravity. The map supports theories of how structure developed in the evolving universe, which has transitioned from a comparatively smooth distribution of matter at the time of the Big Bang. Dark matter filaments began to form first and so provided an underlying scaffolding for the subsequent development of stars and galaxies from ordinary matter (more) (Figure 1).

The presence of dark matter around the Milky Way was first detected by its gravitational effect on the galaxy's rotation curve, the orbital velocity of the observable stars or gas clouds in orbit around the galactic core. Astronomer Vera Cooper Rubin found over decades of radio observations that the rotational velocity of clouds of ionized hydrogen (HII regions) in spiral galaxies like the Milky Way was not decreasing at increasing distance from their galactic cores, like the velocity of the planets around the Sun. Rubin's finding of relatively flat rotation curves far beyond the reach of the visible matter of galaxies is believed to be possible only if the observed galaxies have large amounts of non-luminous mass in their extended haloes (more on Vera Rubin and Dark Matter) (Figure 2).

The Milky Way's dark halo is believed to outweigh the galaxy's normal matter by around a factor of 20 . While the inner edge of the luminous hypothesized outer ring that

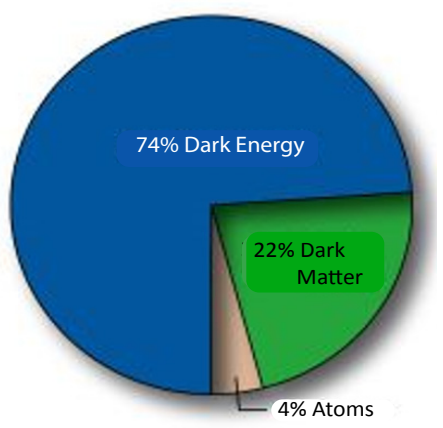

Figure 1: A three-dimensional map of the large-scale distribution of dark matter in part of the universe.

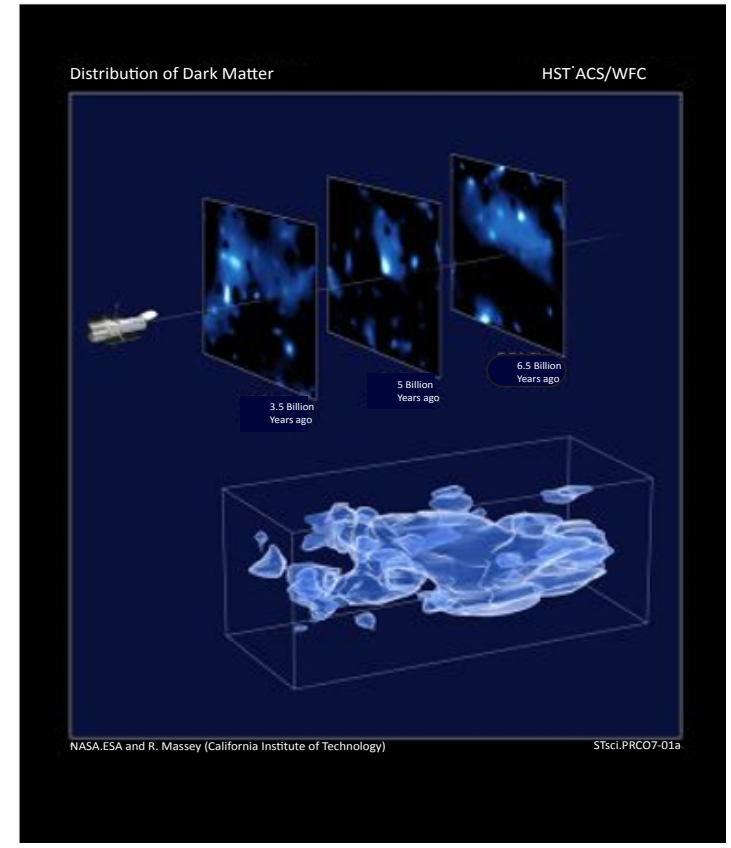

Figure 2: Distribution of matter.

surrounds the spiral disk of the Milky Way may be around 120,000 light-years (ly) across, the dark halo encompasses and permeates even the enormous luminous halo of scattered individual stars and globular clusters, extending some 300,000 to 400,000 ly out from the galactic center in radius (twice that in diameter). In 2006, a team of scientists (including Jürg Diemand, Michael Kuhlen, and Piero Madau) modelled the process by which dark matter "clouds" are attracted to form the Milky Way's dark halo (Diemand et al, 2006). They simulated the development and movement of 234 million "cloudlets" as they come together to form a dark halo about the same size as that around the Milky Way. Their simulations show that there should be at least 10,000 separate "subhaloes" of dark matter within the overall galactic halo, each at least a few thousand light years across. Over time, a fair number of these galactic "seeds" should have attracted ordinary matter (mostly hydrogen and helium gas) to form star clusters. About 120 of the larger clumps of dark matter should have become large enough to have attracted enough gas to become dwarf galaxies, altough astronomers have identified about 15 dwarf satellite galaxies around the Milky Way thus far [16-18].

It is possible that many subhaloes did not form dwarf galaxies because dark matter has some property that prevents it from forming dense clumps. For example, it might be unexpectedly hot, and therefore hard to compress. Many astronomers currently believe, however, that there are other explanations for the paucity of observed satellite companions around the Milky Way. It may be that most of the subhaloes were sterilized by ultraviolet light from the earliest stars, which heated up intergalactic gas so that that it has been more difficult for subhaloes to capture. In addition, supernova explosions may have blasted gas out of many early dwarf galaxies, halting their continued development (Figure 3). 
Astronomers have detected evidence that dwarf satellite galaxies are disturbing the cocoon of dark matter around the Milky Way and causing its disk to warp. In 1957, astronomers surveying galactic hydrogen gas discovered that the Milky Way is not flat but warped near its edges like a fedora hat, with one side of its spiral disk curving as much as 20,000 light years above the main galactic plane and the other dipping a little less below it. While some researchers suspected the warp was caused by the two Magellanic Clouds (nearby satellite galaxies that orbit the Milky Way every 1.5 billion years), subsequent calculations showed they alone were not massive enough to produce the disk's warp. In January 2006, a team of researchers (including Leo Blitz, Martin Weinberg, Carl Heiles, and Evan Levine) announced finding evidence that the Magellanic Clouds can account for the warp but only because their motion around the Milky Way generates a powerful gravitational wake within the massive dark halo. As the Magellanic Clouds orbit the Milky Way, computer simulations indicate that the galactic disk ripples over time and its edges ruffle "like a table cloth in the breeze" (U.C. Berkeley news release; and Maggie McKee, New Scientist, January 10, 2006). On January 9, 2007, astronomers announced that new measurements of the velocities of the Megallanic Clouds through space suggest that the Milky Way's combined dark and visible mass must be twice as much as originally thought if the Clouds are trully orbiting satellites of the galaxy (more).

In January 2010, a team of astronomers (including David Law) announced at the $215^{\text {th }}$ Meeting of the American Astronomical Society that the cloud of dark matter that surrounds our Milky Way galaxy appears to be shaped like a "squashed beach ball" that is oriented perpendicularly to the galaxy's spiral disk. The team studied the path of a dwarf galaxy called the Sagittarius dwarf elliptical or spheroidal galaxy, whose stars have been "shredded" into a long tidal stream as the smaller galaxy was gravitationally drawn into a merger with the Milky Way beginning some three billion years ago. Their hypothesis is that the the gravitional pull of the Milky Way's immense halo of surrounding dark matter should have shaped the trajectory of the tidal stream of stars ripped from the Sagittarius galaxy as it was drawn in.

The Milky Way's halo of dark matter is oriented roughly perpendicularly to the spiral disk in the shape of a squashed beach ball -- Sol's location is markeded by the yellow dot (Figure 4).

Their study of this "debris" stream of stars suggests that the distribution of dark matter around the Milky Way is very different to that of the galaxy's stars and gas matter seen in luminous ordinary matter. Although computer simulations had suggested that the halo should mimick the Milky Way's spiral disk of stars, the team's results indicate that the dark halo is oriented roughly perpendicular to the disk and is distributed roughly half as thick as it is wide. (More discussion is available fromRachel Courtland, New Scientist, January
6, 2009, which includes a video animation describing the proposed shape and orientation of the Milky Way's dark matter; and David Law's description of his current research on Galactic Structure and Dynamics, being undertaken by studying the formation and evolution of tidal streams from Local Group of dwarf spheroidal galaxies).

\section{Should We Worry about Superflares?}

This artist's impression shows a superflare around a distant star. The mature sun may still be prone to temper tantrums. A new study suggests that older stars like the sun can produce superflares - huge bursts of energy visible across hundreds of light-years (Figure 5). Superflares used to be thought of as a younger-star phenomenon, researchers said in a statement about the new study, but the new work suggests it can happen on the sun at rare intervals, of perhaps once every few thousand years. (The sun is about 4.6 billion years old and midway through its lifetime). The sun is hard to predict on even a daily basis, so it's difficult to say when a superflare would occur. However, the new work's lead

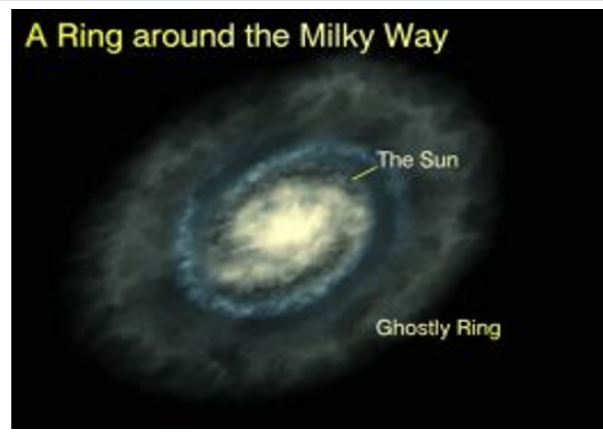

Figure 3: A Ring around the Milky Way.

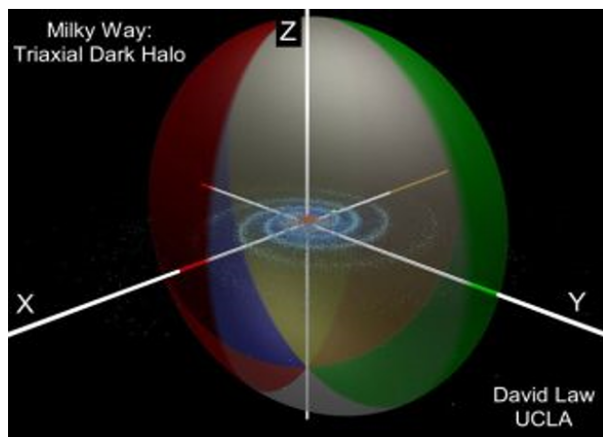

Figure 4: The Milky Way's halo of dark matter is oriented roughly perpendicularly to the spiral disk in the shape of a squashed beach ball -- Sol's location is markeded by the yellow dot.

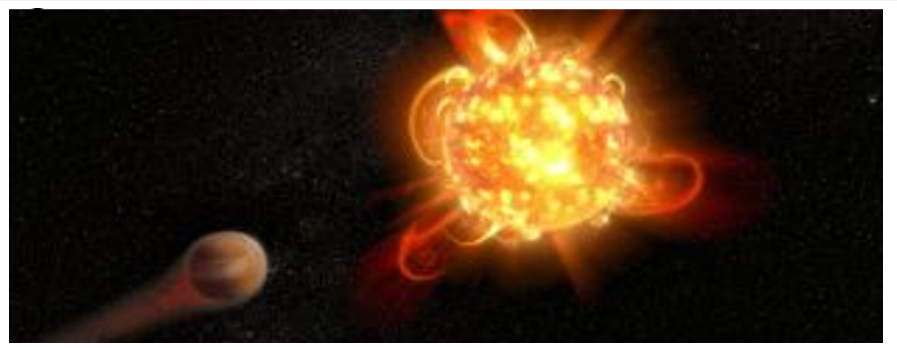

Figure 5: Shows a superflare around a distant star. 
author, Yuta Notsu - a visiting researcher at the University of Colorado Boulder — said this possibility should inspire everyone to beef up electronics against radiation.

"If a superflare occurred 1,000 years ago, it was probably no big problem. People may have seen a large aurora," Notsu said in a statement, referring to the dancing Northern Lights or Southern Lights produced by solar particles interacting with molecules of Earth's atmosphere. "Now, it's a much bigger problem because of our electronics". We already know the power of the sun can knock out power lines, electronics and satellites. Coronal mass ejections from the sun - or large plumes of charged particles - have caused issues with our infrastructure in the past, such as the extraordinary 1859 Carrington Event superstorm that affected telegraph communications. A superflare, however, would be worse. The superflare would be hundreds or thousands of times more powerful than the most active solar flares recorded $[16,17]$. "If a superflare erupted from the sun ... Earth would likely sit in the path of a wave of high-energy radiation. Such a blast could disrupt electronics across the globe, causing widespread blackouts and shorting out communication satellites in orbit," representatives from the University of Colorado Boulder said in the statement.

The new superflare data came from NASA's Kepler space telescope, which looked for planets at faraway stars between 2009 and 2018. While looking for new worlds, Kepler also saw a lot of star activity. It spotted a few superflares, moments when the starlight would suddenly get brighter before dimming again. Curious about Kepler's findings, the researchers looked to the European Space Agency's Gaia spacecraft - which studies stellar movements and brightnesses across a billion stars - and the Apache Point Observatory in New Mexico. The two telescopes saw 43 superflares that came from stars similar in age and size to our own sun, the study's researchers said. Statistics from their data show that most superflares do come from younger stars, which can flare about once a week. And our own sun is still prone, but just once every few thousand years. Notsu presented his research Monday (June 10) at the $234^{\text {th }}$ meeting of the American Astronomical Society in St. Louis. The results were also detailed May 3 in The Astrophysical Journal [6].

\section{New, fifth force of nature}

There may be a fifth force of nature, a new study suggests.

"If true, it's revolutionary," study lead author Jonathan Feng, a professor of physics and astronomy at the University of California, Irvine, said in a statement. "For decades, we've known of four fundamental forces: gravitation, electromagnetism, and the strong and weak nuclear forces," Feng added. "If confirmed by further experiments, this discovery of a possible fifth force would completely change our understanding of the universe, with consequences for the unification of forces and dark matter." [8 Baffling Astronomy
Mysteries]. The Hungarians detected tantalizing evidence of a previously unknown particle just 30 times heavier than an electron - a result they published early this year.

“The experimentalists weren't able to claim that it was a new force," Feng said. "They simply saw an excess of events that indicated a new particle, but it was not clear to them whether it was a matter particle or a force-carrying particle". The new work by Feng and his team suggests that the Hungarians found not a "dark photon" but rather a "protophobic X boson" - a strange particle whose existence could indicate a fifth force of nature. The known electromagnetic force acts on protons and electrons, but this newfound particle apparently interacts only with electrons and neutrons, and then only at very short distances, researchers said. "There's no other boson that we've observed that has this same characteristic," co-author Timothy Tait, also a professor of physics and astronomy at UCIrvine, said in the same statement. "Sometimes we also just call it the 'X boson,' where 'X' means unknown".

The potential fifth force maybelinked to the electromagnetic and strong and weak nuclear forces, as "manifestations of one grander, more fundamental force," Feng said. It's also possible that the universe of "normal" matter and forces has a parallel "dark" sector, with its own matter and forces, Feng added. "It's possible that these two sectors talk to each other and interact with one another through somewhat veiled but fundamental interactions," Feng said. "This dark-sector force may manifest itself as this protophobic force we're seeing as a result of the Hungarian experiment. In a broader sense, it fits in with our original research to understand the nature of dark matter."

While such speculation is intriguing, the researchers stressed that their interpretations are preliminary, and that further study and experiments are needed. Indeed, particles supposedly observed in accelerators sometimes turn out to be statistical flukes. The good news is that many scientists should be able to do the required follow-up work, Feng said $[18,19]$. "Because the new particle is so light, there are many experimental groups working in small labs around the world that can follow up the initial claims, now that they know where to look," he said [20].

Why the Universe shouldn't exist at all. Don Lincoln, a senior physicist at Fermilab, does research using the Large Hadron Collider. He is the author of "The Large Hadron Collider: The Extraordinary Story of the Higgs Boson and Other Stuff That Will Blow Your Mind", and produces a series of science education videos. To the question: Why is there (in our Universe) something including cancer, rather than nothing? He is giving the simplest scientific answer: We shouldn't exist at all. Give some scientists 65 pounds of rare isotope of germanium, cool it to temperatures cold enough to liquefy air, and place their equipment nearly a mile underground in an abandoned gold mine, and you'll have the beginnings of an answer. Their project is called the Majorana 
Demonstrator and it is located at the Sanford Underground Research Facility, near Lead, South Dakota.

To grasp why science has trouble explaining why matter exists - and to understand the scientific achievement of Majorana - we must first know a few simple things. First, our Universe is made exclusively of matter, all people, the Earth, even distant galaxies. All of it is matter [20,21]. Our best theory for explaining the behavior of the matter and energy of the Universe contradicts the realities that we observe in the Universe all around us. This theory, called the Standard Model, says that the matter of the Universe should be accompanied by an identical amount of antimatter, which, as its name suggests, is a substance antagonistic to matter. Combine equal amounts of matter and antimatter and it will convert into energy. And the street goes both ways: Enough energy can convert into matter and antimatter (since antimatter's discovery in 1931). Modern cosmology says the Universe began in an unimaginable Big Bang - an explosion of energy. In this theory, equal amounts of matter and antimatter should have resulted in cancer. So how our Universe made exclusively of matter? However, we don't know the process whereby the asymmetry in the laws of the Universe arose. One possible explanation revolves around a class of subatomic particles called leptons.

The most well-known of the leptons is the familiar electron, found around atoms. A less known lepton is called the neutrino. Neutrinos are emitted in a particular kind of nuclear radiation, called beta decay. It occurs when a neutron in an atom decays into a proton, an electron, and a neutrino. Neutrinos are fascinating particles. They interact extremely weakly, a steady barrage of neutrinos from the nuclear reactions in the Sun pass through the entire Earth essentially without interacting. Still a mystery to scientists is whether there is a difference between neutrino matter and neutrino antimatter. While we know that both exists, we don't know if they are different subatomic particles or if they are the same thing. We don't know which kind of twins the neutrino matter/antimatter pair are. If neutrinos are their own antimatter particle, it would be an enormous clue in the mistery of missing antimatter and proliferation of matter in cancer [22-25].

The way they do that is to look first for a very rare form of beta decay, called double beta decay. That's when two neutrons in the nucleus of an atom simultaneously decay. If neutrinos are their own antiparticle, an even rarer thing can occur called HYBRID neutrinoless double beta decay (the term: Robert Skopec). In this process, the neutrinos are absorbed before they get outside of the nucleus. In this case no neutrinos are emitted. The observation of a single, unambiguous neutrinoless double beta decay would show that matter and antimatter neutrinos were the same. If indeed neutrinoless double beta decay exists, it's very hard to detect and it's important that scientists can discriminate between the many types of radioactive decay that mimic that of a neutrino. This requires the design and construction of very precise detectors. So that's what the Majorana Demonstrator scientists achieved. Once and for all, it can answer the question of whether matter and antimatter neutrinos are HYBRID: the same or different. With that information in hand, it might be possible to understand why our Universe is made of matter leading to cancer too [10].

\section{HYBRID majorana fermion change the world}

Chinese scientists won a major victory, by proving that the Majorana fermion - a particle we've found tantalizing hints of for years - genuinely exists. This discovery has huge implications for quantum computing of cancer, and it might change the World. A Majorana fermion is weird even by the standards of quantum physics. The Majorana fermion doesn't have a charge, which allows the mystery of cancer to be HYBRID: matter and anti-matter at the same time! The fact that it doesn't have a charge, and also happens to be the exact reverse of itself at the same time. Quantum computers of cancer, are like a huge pile of dimmer switches. You can set these dimmers much, much faster than you can flip on this light switches, because the dimmers are all wired to each others, immediately as tumors. These dimmers, e. i. quantum bits, are what's called entangled in cancer. If you change one quantum bit, the others it's entangled with change with it, even if they're a million miles away from each other. That's where Majorana fermions as metastasis come in due to their HYBRID: no-charge nature [18].

\section{References}

1. Bartels M. Newsweek. 2018.

2. Darimont $\mathrm{CT}$, Fox $\mathrm{CH}$, Bryan $\mathrm{HM}$, Reimchen TE. The unique ecology of human predators. Science. 2015; 349: 858-860.

PubMed: https://www.ncbi.nlm.nih.gov/pubmed/26293961

3. Dolev Sch. The quantum computing appocalypse is imminent. Tech Chrunch. 2018.

4. Gibbons D. General proof and method of sustained state management in autonomous systems. Personal communications. 2018; 5.

5. Gold JI, Shadlen MN. The Neural Basis of Decision Making. Annu Rev Neurosci. 2007; 30: 535-574.

PubMed: https://www.ncbi.nlm.nih.gov/pubmed/17600525

6. Howell E. Our Aging Sun Is Still Capable of Unleashing 'Superflares.' Should We Worry? Science \& Astronomy. 2019.

7. Hsu M, Kraibich I, Zhao C, Camerer CF. Neural Response to Reward Anticipation Under Risk Is Nonlinear in Probabilities. J Neurosci. 2009; 29: 2231-2237.

PubMed: https://www.ncbi.nlm.nih.gov/pubmed/19228976

8. Kourtidis A, Ngok SP, Anastasiadis PZ. Distinct E-cadherin-based complexes regulate cell behavior through miRNA processing or $\mathrm{Src}$ and p120 catenin activity. Nat Cell Biol. 2015; 17: 1145-1157. PubMed: https://www.ncbi.nlm.nih.gov/pubmed/26302406

9. Lin J, Marcolli M, Ooguri H, Stoica B. Tomography from Entanglement. 2014.

10. Lincoln D. 2018.

11. Lindsey J. So...What Is Dark Matter Anyway? Popular Mechanics, May 31, 2019 Mayo Clinic. (2015) Discovery of new code makes reprogramming of cancer possible. 
12. Masterson A. Multiverse theory cops a blow after dark energy findings. Cosmos. 2018.

13. Medrano K. Physics: Scientists Rewrite Quantum Theory to Do the Impossible Track Secret Particles. 2017.

14. Paulus MP, Frank LR. Anterior cingulated activity modulates nonlinear decision weight function of uncertain prospects. Neuroimage. 2006; 30: 668-677.

PubMed: https://www.ncbi.nlm.nih.gov/pubmed/16321546

15. Prigogine I. The End of Certainty. Time, Chaos, and the New Laws of Nature. First Free Press Edition. 1997; 161-162.

16. Ramachandran VS, Hubbard EM. Psychological Investigation into the Neural Basis of Synesthesia. Proceedings of the Royal Society of London. 2001; 268: 979-983.

17. Rechtsman M. Penn State Univesity. Nature. 2018.

18. Seitz D. 2018: https://www.yahoo.com/news/majorana-fermiongoing-change-world-185818775.html
19. Siegl E. https://www.forbes.com/sites/startswithabang/2018/01/10/ new-dark-matter-physics-could-solve-the-expanding-univrse-controversy/\#5

20. Wall M. Science \& Astronomy 2016.

21. University of California - Berkeley. "Recording a thought's fleeting trip through the brain: Electrodes on brain surface provide best view yet of prefrontal cortex coordinating response to stimuli." 2018.

22. Skopec R. I. An Explanation of Biblic Radiation: Plasma. Journal of Psychiatry and Cognitive Behavior. 2017.

23. Skopec R. II. Artificial hurricanes and other new Weapons of Mass Destruction. International Journal of Scientific Research and Management. 2018; 7751-7764.

24. Skopec R. III. Intelligent Evolution, Complexity and Self-Organization. NeuroQuantology. 2015; 13: 299-303.

25. Skopec R. IV. Translational Biomedicine and Dichotomous Correlations of Masking. Translational Biomedicine. 2016; 1: 47. 